\title{
Coulisses
}

Revue de théâtre

12 | Printemps 1995

Varia

\section{Mythe et dramaturgie dans Partage de Midi : l'expression du sacré et du divin}

\author{
Jacques Houriez
}

\section{(2) OpenEdition}

\section{Journals}

Édition électronique

URL : http://journals.openedition.org/coulisses/3294

DOI : $10.4000 /$ coulisses.3294

ISSN : 2546-9460

Éditeur

Presses universitaires de Franche-Comté

\section{Édition imprimée}

Date de publication : 1 mai 1995

Pagination : 30-36

ISSN : 1150-594X

\section{Référence électronique}

Jacques Houriez, «Mythe et dramaturgie dans Partage de Midi : l'expression du sacré et du divin »,

Coulisses [En ligne], 12 | Printemps 1995, mis en ligne le 15 mars 2019, consulté le 23 octobre 2019

URL : http://journals.openedition.org/coulisses/3294 ; DOI : 10.4000/coulisses.3294

Ce document a été généré automatiquement le 23 octobre 2019.

Coulisses 


\title{
Mythe et dramaturgie dans Partage de Midi : l'expression du sacré et du divin
}

\author{
Jacques Houriez
}

Ce n'est pas la faute du cuivre, disait Rimbaud, s'il s'éveille clairon. À la fin du poème " Aube ", l'enfant sort du sommeil. L'expérience poétique est ainsi vécue comme une extase, un moment privilégié hors de la vie quotidienne. Et Claudel, fidèle à la pensée de son père spirituel, la précise par la bouche des poètes Ly et Cœuvre de La Ville :

...je ne parle pas selon ce que je veux, mais je conçois dans le sommeil.

Et je ne saurais expliquer d'où je retire ce souffle, c'est le souffle qui m'est retiré.

Dilatant ce vide que j'ai en moi, j'ouvre la bouche.

Et, ayant aspiré l'air, dans ce legs de luimême par lequel l'homme à chaque seconde expire l'image de sa mort, Je restitue une parole intelligible. $\mathrm{Et}$, l'ayant dite, je sais ce que j'ai dit. ${ }^{1}$

2 C'est ici l'image du sommeil créateur, la

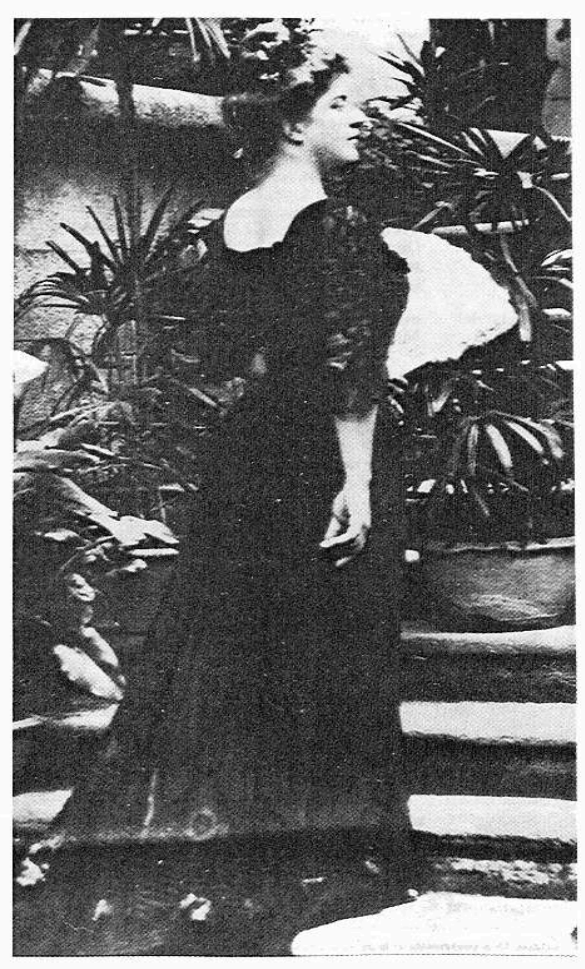
mort momentanée d'Adam qui permit la naissance d'Ève. Mais elle suppose une expérience proche de celle de Rimbaud. Le Poète, le créateur s'exprime dans un état si fondamentalement différent de la vie quotidienne, les réalités des deux vécus sont l'une à l'autre si étrangères que l'écrivain ne découvre sa propre parole qu'une fois sorti de l'état poétique. 
Et pourtant, bien qu'il y ait, tous deux inédits, un journal des années vécues avec Rosalie Vetch et une correspondance, lorsque Jean Amrouche demande au dramaturge vieilli des précisions sur le vécu de son drame du Partage de Midi, celui-ci le renvoie à la pièce. Elle dit tout, il n'y a rien à ajouter. Bien plus, il affirme dans sa correspondance avec Jean-Louis Barrault que c'est Eve Francis dans le rôle d'Ysé, c'est la représentation de la pièce qui lui a fait comprendre, enfin, quarante années après, ce qui lui était arrivé. Ainsi refuse-t-il toute dichotomie qui maintiendrait définitive la scission entre le créateur et l'homme du quotidien, entre la réalité vécue et la réalité créée. Il y oppose un paradoxe : l'univers du créateur est totalement étranger à celui de tous les jours, mais il en détient la vérité. Il n'est même qu'une seule vérité, celle de la création littéraire. La parole poétique peut seule révéler la réalité profonde du vécu quotidien. La vérité du drame n'est donc pas de l'ordre de l'événement. Elle n'est pas de l'ordre du récit, elle ne relève pas du parler quotidien. Elle emprunte celui de l'imaginaire, parfaitement élucidé, d'ailleurs par M. Moriaki Watanabé. Les écrits intimes, quelques aveux, les thèmes, les lieux de l'action nous invitent à privilégier un triple langage mythique, celui d'une fatalité extérieure, d'abord : un rituel sacrificiel païen traduit la plongée dans un absolu destructeur. Perdus sur un bateau, « miette mouvante » au sein de l'Océan indien, les personnages sont entraînés inexorablement vers un destin qui les écrase. $\mathrm{Au}$ second acte, la tombe en oméga où les amants se donnent rendez-vous les enserre dans un étau de mort. Ils sont livrés à la passion comme à un feu intérieur qui achève de les anéantir. Ils n'ont pas supporté l'affrontement avec le sacré. Il ne faudra pas moins que la mort, enfin, dans la nuit d'une liturgie mystique, pour libérer Mesa du Moi farouchement égoïste où il s'était enfermé et le livrer à l'absolu du divin, le véritable objet de son désir. Encore fera-t-il clairement l'aveu qu'il ne sait toujours se donner ni recevoir et encore moins assumer la charge de la femme.

\section{L'absolu destructeur du rituel païen}

3 Passé Suez... Un poème de Kipling, «Mandalay », évoque le passage de Suez : "Ship me somewheres east of Suez, where the best is like the worst, / Where there aren't no Ten Commandments an'a man can raise a thirst. » Claudel l'évoquera dans « Le Livre sur la Chine». C'est aussi un thème essentiel de Partage de Midi. Passé Suez, on a laissé derrière soi la famille, le pays, l'Europe avec ses lois. C'est une plongée hors du social, dans la pureté de l'être. Et, si l'union avec l'absolu divin doit avoir lieu dans la nuit, le premier contact se fait dans un plein soleil, une lumière, une chaleur qui sont d'abord destruction et écrasement. On plonge d'un seul coup en pleine mythologie érotique et païenne. «La mer à l'échine resplendissante » - On croirait entendre Eschyle - est " comme une vache terrassée que l'on marque au fer rouge $"^{2}$. La première image que Mesa donne de l'acte amoureux est donc marquée de violence sadique. La féminité est humiliée. Le bourreau qui la sacrifie et, en guise de baisers, plonge des couteaux dans son flanc, n'est pas le mâle, le taureau. Il est le plus cruel des dieux, Baal, celui des fours où l'on immole les humains. Et la mer est un «miroir». Elle ne subit pas, mais réverbère et renvoie les couteaux qu'elle reçoit. L'amante elle aussi sacrifie. Détruite, elle est aussi destructrice. L'amant est tout aussi bien qu'elle aboli, sacrifié et humilié. La première représentation de l'acte amoureux est ainsi dès l'abord charnellement païenne, mystique et tragique. Elle est d'autant plus pour les héros l'annonce de la mort qu'ils se trouvent à l'intérieur du four, entre le miroir de la mer et le soleil-Baal, doublement lardés par les couteaux reçus et renvoyés. Ils sont livrés au-dessus d'eux à 
un absolu mâle de passions déchaînées, et en dessous, à cette mer femelle, sans « forme, sans couleur, pure, absolue [elle aussi], énorme, fulminante $»^{3}$, eaux sacrées que souille leur navire, où ils sont perdus hors du temps, hors du lieu, entraînés vers un destin inconnu, mais qu'ils savent tragique.

Ce ne sont là, cependant, dirait l'Empereur du Repos du Septième Jour, que vaines images, et nous comprenons vite que ces personnages sont d'abord écrasés par un destin intérieur. Ysé et Mesa sont brisés par l'échec de leur vie : ils ont voulu, mais n'ont pas su aimer.

Lorsque, par un jeu métonymique, le foyer ardent du soleil devient, dans la bouche de Mesa, l'image du foyer domestique: "Le voilà, notre foyer, troupe errante! Ne le trouvez-vous pas allumé comme il faut? " ${ }^{4}$, Ysé est la première à faire devant lui l'aveu de sa faiblesse : «Il me tue ! Je ne puis en supporter la force. » Avoir pour seul foyer l'astre dévorant, c'est être fils du soleil, c'est jouir d'une liberté dont l'homme seul qui n'a pas de liens peut se réjouir: "Vous autres, vous êtes libres! Mais moi, pauvre femme, avec ces trois enfants dans mon tablier, quatre membres chacun! $»^{5}$. Pour elle, le soleil ne peut être qu'un dieu destructeur.

En fait, des quatre protagonistes, Amalric est le seul qui se réjouisse de cette liberté et ne sente pas peser sur lui la main de la mort. De Ciz, l'époux d'Ysé, est «amer d'avoir fini d'être jeune ", et Mesa fait l'éloge du soir et du saint qui triomphe à son dernier jour, annonçant à son insu et par une sorte d'ironie tragique le sort qui l'attend. Il ne trouvera le salut que dans le soir, la nuit et la mort.

Ainsi livrés à l'absolu et dépouillés d'eux-mêmes, les deux protagonistes principaux ont l'intuition de leur vérité propre qui est de vouloir et de ne savoir aimer Tous deux ignorent ce qu'est l'amour et, malgré les trois «poulains » qu'elle a enfantés, Ysé est aussi vierge de ce sentiment que Mesa lui-même. Les premières héroïnes claudéliennes ne découvrent leur vocation qu'en une sorte d'état second caractérisé par la danse, comme la Belle Dame de La Divine Comédie. Déjà la Princesse de Tête d'Or avait revêtu un vêtement liturgique pour le connaître. Et, dans le dernier moment, Ysé rejoindra Mesa "vêtue de blanc en état de transe hypnotique " ${ }^{6}$. Pour l'instant, elle rêve au sommeil d'Adam, c'est-à-dire à sa vraie naissance qui sera d'aimer. Pour cela, il ne faut pas comprendre,

il faut perdre connaissance. Moi, je suis trop méchante, je ne puis pas.

C'est une opération à subir. C'est le tampon d'éther que l'on vous fourre sous votre nez.

Le sommeil d'Adam, vous savez! c'est écrit dans le catéchisme. C'est comme ça que l'on a fait la première femme.

Une femme, dites, songez un peu! tous les êtres qu'il y a en moi ! Il faut se laisser

faire.

Il faut mourir

Entre les bras de celui qui l'aime, et est-ce qu'elle se doute, l'innocente.

Rien du tout ! ce qu'il y a en elle et ce qu'il en va sortir? Elle ne sait rien ! une mère de femmes et d'hommes! ${ }^{7}$

Ce qui devrait en sortir, c'est, bien sûr, la femme véritable, Eve, l'Amante, la Mère des hommes... et de Mesa. Mais, pour l'instant, Ysé ressent comme irrémédiable l'échec de son mariage. La vie l'a usée.

Mesa non plus n'est pas encore né à l'amour. Il en est incapable. Ysé le lui dit sans ménagement : il ne s'occupe que de soi et sait s'offrir, mais non se donner. Il revient de France où il s'est proposé à Dieu, mais n'a pu se donner. Il a été trouvé manquant. Pour lui, aimer, c'est atteindre dans un don total cet absolu qui lui a été refusé. Repoussé par 
Dieu, il s'adresse à la femme. Dans un élan plein de contradictions et d'ambiguïtés, il va le rechercher dans une passion charnelle, violente, qui sera incapable de le lui donner.

6 Cette première rencontre se termine de façon ambiguë : chacun accepte que l'autre se donne : «Ah ! semble promettre Ysé à Mesa, qui se donne comme il faut, il forcera bien qu'on l'accepte! ", tandis que Mesa devant la mer sous la lumière du soleil couchant songe aux yeux d'une femme qui s'abandonne. Ils semblent prêts l'un et l'autre à recevoir, mais à recevoir seulement.

Le sentiment d'être livré seul à une fatalité païenne violemment hostile et irrationnelle, d'être inférieur à sa vocation, avait été exprimé par les premières œuvres, L'Endormie dont le Poète vivait dans un cauchemar perpétuel, Une Mort prématurée où Marie n'avait su aimer et s'était livrée, avec le Frère à un amour adultère et incestueux, où le mari trompé, Henri, était déjà livré à la mort. L'arrivée de Claudel en Chine, ses Agendas en témoignent, puis l'échec de la vocation sacerdotale, l'ont avivé, lui ont donné une dimension toute nouvelle. Et le drame qu'il a vécu l'a plongé dans un véritable désespoir. La première vérité de Partage de Midi, comme celle des premiers drames, n'est donc pas de l'ordre de l'anecdote, mais de la vie spirituelle. Elle est dans un sentiment d'écrasement, d'abandon et de désespérance.

\section{La possession de l'interdit.}

7 Les deux amants ont découvert leur vérité dans leur anéantissement. Leur union achève leur destruction. Précédée par l'intuition de la mort, celle-ci se réalise dans le sentiment du néant et d'une culpabilité qui va jusqu'à la notion de meurtre.

Certes, Ysé et Mesa découvrent chacun la valeur de l'autre. Lui, dans un rappel du Cantique, voit en Ysé une colonne, une puissance, une mère et une sœur ${ }^{8}$ et, dans un souvenir païen, retrouve un thème solaire qui réintroduit l'évocation de la colonne9.

De son côté, Ysé, dans un souvenir de l'Ecclésiaste cette fois, rappelle que la folie de son amant peut être sagesse puisqu'elle est celle de l'amour ${ }^{10}$.

Par contre, elle reçoit Mesa dans ses bras avec un «Pauvre enfant! Mesa! Pauvre Mesa! $»^{11}$, et le « Tout est fini » de ce dernier qui rappelle le « Tout est consommé » de Jésus en croix, exprime un véritable désespoir. Comme Tête d'or, en effet, il est un homme de désir, il aspire au bonheur. Mais son désir qu'il eût voulu de Dieu n'a pu être que de la femme. Tête d'Or, Louis Laine avaient refusé le piège de la Mère. Lui, est tombé dans celui de la femme maternelle et interdite: "Et qui dit que tu es le bonheur? ah, tu n'es pas le bonheur! tu es cela qui est à la place du bonheur! $»^{12}$. L'amour l'a livré au pouvoir de l'Autre et l'a anéanti.

Claudel, dans sa préface, cite Ezéchiel : "Je ferai sortir de toi un feu qui te dévorera. » Et c'est sous le signe du feu intérieur que Mesa, effectivement, se donne à Ysé, «le feu que fait un cœur qui s'embrase ", le feu de la création, les laves de la terre à son premier moment.

Il l'a accueilli avec une théâtralité, un recul par rapport à soi-même qui n'exclut pas la sincérité : «(récitant:) «Au moins je souffre, au moins je suis très malheureux. » »" Ysé, qui est polonaise et en cela proche peut-être de la première femme aimée par Claudel, des futures Fausta de La Cantate à trois voix et Lûmir de L'Otage, a eu l'intuition, et, plus explicitement que son amant, le désir de la mort. Sa vérité est aussi de mourir, mais dans un grand éclair comme elle en fera le choix final. Pour l'instant, elle veut se perdre en l'homme, être mangée comme une mangue, dans un grand élan de sensualité. 
Et, lorsque Mesa, dans le souvenir d'Ézéchiel et de la Genèse voit en elle la violence d'une âme qui brûle et d'une terre en création, elle ne peut que répondre : «Ah, je suis bien vaincue ${ }^{14}$. Pour tous deux, l'amour est l'abolition de l'être. Ils se savent dévorés par le feu d'une passion qu'ils ne peuvent assumer parce qu'ils ne savent pas aimer.

La notion de culpabilité se greffe immédiatement sur ce sentiment. La source n'en est pas seulement religieuse puisque c'est l'agnostique Ysé qui l'affirme avec le plus de force en rappelant qu'elle est interdite et en pleurant sur le malheur de ses "pauvres enfants ». C'est pourtant à travers un imaginaire biblique que la faute s'affirme, l'histoire de David et Bethsabée et le baiser de Judas. David a provoqué la mort d'un de ses officiers, le Hittite Urie après avoir engrossé sa femme. Ainsi Ysé incite-t-elle son amant à faire mourir De Ciz en le lançant dans une entreprise meurtrière, ce qu'il fait avec beaucoup d'habileté. Mais la dernière image qu'elle gardera à l'esprit sera celle du cadavre de son mari dans une paillote misérable, évocation accompagnée, bien sûr, de celle des " pauvres enfants ». La faute des amants est double, adultère et meurtre. Mesa $\mathrm{y}$ ajoute la trahison de Judas. Il guide vers la mort un homme qui voit en lui « un bon, un sincère ami ", à qui il prend la main et de qui Ysé exige un baiser. Mais, si l'Ancien Testament dit la colère de Dieu, le repentir de David et le pardon, Mesa, au moment même de sa mort, regardera en face l'agneau terrible, lui demandera de ne pas faire le Dieu avec lui, et s'il parle de péché, c'est d'égoïsme qu'il s'accusera. Pour le reste il est plutôt la victime d'une passion irrépressible dont il ne se sent pas responsable et qui, en soi, n'a rien de condamnable. Il envisagera moins la notion chrétienne de faute qui prend en compte essentiellement le tort fait à l'autre et à soi-même, que la notion prométhéenne de rupture de l'interdit. Il reste, comme Tête d'Or, Prométhée face à Zeus, c'est pourquoi nous demeurons, en fait, dans un contexte païen, dans le domaine du sacré, non du divin.

Ce langage de mort qui fait d'Amalric le seul survivant de cette saga de la passion charnelle est en totale contradiction avec la réalité événementielle. Il n'y a eu, en fait, ni assassinat ni trahison, bien au contraire. L'enfant né de Paul et de Rosalie, une fille, d'ailleurs et non un garçon, est toujours vivant à l'âge de 90 ans. Paul et Rosalie euxmêmes ont longuement tardé à mourir. Mais il traduit la lassitude et le sentiment de déréliction devant l'échec de la grande passion désirée, celui d'avoir été manquant à l'amour de la femme après l'avoir été à Dieu.

La passion charnelle a contraint Mesa, comme Claudel, à sortir de soi. Elle ne lui a permis ni d'aimer, ni d'accepter l'amour de l'autre.

\section{La réalisation dans la nuit}

Du plein soleil, le drame est passé au soir, puis à la nuit, mais une nuit qui est le lieu de la réalisation, non de la damnation comme dans le premier théâtre claudélien. Ysé a quitté Mesa, et le soir reçoit deux vaincus. C'est, en la personne d'Ysé, d'une femme détruite que s'est emparé Amalric, son second amant.

Mesa n'est pas moins brisé. Lorsqu'il vient libérer Ysé assiégée et promise avec Amalric à la mort, il refuse de regarder la réalité en face et choisit le malentendu : il paraphrase les paroles du père vers qui revient l'enfant prodigue : «Parce que je t'avais perdue et voici que je t'ai retrouvée ! $»^{15} \mathrm{En}$ fait, il n'est pas dans la situation du père. Il n'est pas non plus comme le berger qui retrouve la brebis égarée. Ysé ne revient pas à lui et n'est pas repentante. Elle ne lui répond que par le silence, comme si, à la vie avec lui, elle 
préférait la mort avec Amalric.

Vaincu dans un bref combat avec ce dernier, dépouillé du salut qu'il apportait, abandonné à la mort, le héros pénètre alors dans la nuit qui est, pour les deux amants, le moment d'une nouvelle vérité. La passion dans le grand soleil d'août et celle du soir les avaient rapprochés sans pouvoir les unir. De même, la nuit mystique est à la fois un trait d'union et un écran. Mesa y découvre la voie que le plein soleil lui avait interdit de voir. Il découvre le divin et obtient d'en être accueilli. Mais il ne peut toujours se donner. Il envisage plutôt une union dans la séparation telle que celle de Rodrigue et Prouhèze du Soulier de satin.

Comme les autres héros claudéliens, il veut rejoindre dans la mort le Père et l'Absolu. Mais, alors que pour Tête d'Or, le Père était le dieu Soleil et qu'il s'unissait à lui en repoussant l'union avec la femme, Mesa s'unit à la nuit, et non plus avec une divinité païenne, mais avec le Dieu d'Abraham. Et il le fait après avoir cédé devant la femme, après avoir accepté sa maternité.

Bien plus, alors que Tête d'Or épousait directement le soleil, son sang allant au-devant du sien, Mesa a pour intermédiaires « les puissants astres, pareils à de grandes vierges flamboyantes $»^{16}$ dans la nuit mystique. Ils ont vite fait de devenir le cortège des saints dont il reçoit l'intercession :

Mais déjà les portes du Ciel

Se rompent et l'armée de tous les Saints, portant des flambeaux dans leurs mains,

S'avancent à ma rencontre, entourant l'Agneau terrible. ${ }^{17}$

10 Il vit en pécheur l'Apocalypse avec la révélation de l'Agneau vengeur. Mais en pécheur secouru et sûr de lui, il le reçoit comme dans la communion. Il parle directement comme Job au Dieu de terreur, avec confiance et même hardiesse.

Il n'est pas seul à être entré dans une nuit qui révèle à chacun sa propre vérité. Ysé vient l'y rejoindre. Elle revient partager sa mort. Elle apporte à ce spirituel sec et égoïste quelque chose de plus que la chair qui n'avait su les unir, la chair de la chair, dira Claudel, grâce à quoi il pourra sortir de lui-même. Aussi consentent-ils l'un à l'autre. Mesa peut se donner entièrement. Il se donne comme le Christ dans l'Eucharistie : « ...je consens à toi, Ysé ! Voyez, mon Dieu, car ceci est mon corps! » Ysé s'est donnée totalement, elle aussi dans une sorte d'eucharistie, puisqu'il la boit en ce monde comme il la boira en l'autre.

Mais, bien que pour eux tout soit ainsi consommé et qu'il l'ait reçue comme son âme, un abîme aussi les sépare. Mesa la reçoit avec une restriction aussi grave que mystérieuse, puisqu'elle correspond au finale d'Une Mort prématurée où la femme, Marie, s'était rendue coupable d'un adultère incestueux. Ysé est devant Mesa comme Marie devant Henri :

Adieu ! je t'ai vue pour la dernière fois.

Par quelles routes longues, pénibles.

Distants encore que ne cessant de peser

l'un sur l'autre, allons-nous

Mener nos âmes en travail!

Souviens-toi, souviens-toi du signe $!^{18}$

11 Le signe qui, dans Une Mort prématurée n'était pas précisé, est ici clairement désigné. Ce sont pour Ysé les grands cheveux dans le vent, souvenir que l'on retrouve dans la $1^{\text {ère }}$ Ode, symbole de splendeur et de passion charnelle, ici «déchaînés dans le vent de la Mort ». Il est pour Mesa, au contraire, tout spirituel, c'est « L'esprit vainqueur dans la transfiguration de Midi». Mesa rejoint le cortège des étoiles avec la force d'une 
"grande flamme fulminante». Son signe est la marque de l'Esprit, alors qu'Ysé est demeurée charnelle dans cette nuit mystique.

Mais ce qui les sépare est bien plus important. Tout au long de la pièce, ils se sont présentés l'un à l'autre en prononçant le «C'est moi", l'Adsum des prophètes de l'Ancien Testament et des diacres lors de leur consécration. C'était la reprise du thème du nom d'Une Mort prématurée. C'était la promesse aussi de satisfaire à leur vocation qui était de se donner. Ysé n'avait su le faire dans le mariage, ni Mesa par le sacerdoce. Son égoïsme avait été plus fort que son désir. L'échec de leur amour, Ysé le dit ${ }^{19}$ et Mesa le reconnait ${ }^{20}$ vient de ce qu'il n'a pas su se donner ${ }^{21}$. Sans doute n'est-ce pas sans quelque humour qu'après avoir appelé Ysé dans la nuit de son cantique, il se plaint de sa présence : «Je ne puis donc me débarrasser de cette Ysé ? Il ne m'est pas possible / De me défaire de ces deux mains de femme à mes flancs? $\|^{22}$ Mais ce n'est pas non plus sans quelque sincérité. Et si plus tard, il consent à elle en une union pour la mort, et sans doute au-delà, cette union supposera une distance excluant la possession au profit du désir. "Distants encore que ne cessant de peser l'un sur l'autre », les deux amants demeureront ainsi qu'en leur vie unis et séparés, comme tous les couples du théâtre claudélien. C'est le désir et non la possession qui les tiendra unis dans la mort comme ils l'ont été dans la vie, ce désir que Claudel envierait à l'enfer s'il cessait d'exister avec Dieu $^{23}$. Et si lui-même et ses personnages ont tendu toujours passionnément vers Dieu et vers la Femme sans jamais les étreindre, s'ils n'ont pas voulu satisfaire leur besoin, c'est pour le faire toujours plus grand. Seuls dans sa dernière pièce, L'Histoire de Tobie et de Sara, ses nouveaux Animus et Anima se rejoindront en Dieu, mais ce sera la fin du drame claudélien. Le théâtre laissera la place au commentaire biblique.

\section{Les révélations de Partage de Midi.}

12 La difficulté d'aimer, de se donner est une constante de la confession de Claudel. Il en a connu, dès le moment de sa conversion, l'évidence très forte. Pourquoi, en ce cas, lui at-il fallu attendre la première représentation de la pièce pour réaliser ce qui lui était arrivé ?

Proust, dans son Contre-Sainte-Beuve, nous dit que le créateur n'a rien à voir avec l'homme de tous les jours. Si ce dernier est chez Claudel celui qui s'exprime par la confession directe, il nous dit, dans "Ma conversion", sa haine des prêtres et son exécration des fidèles restées intactes après le contact mystique. Il met alors son refus de l'Autre sur le compte de son éducation. La faute en incombe à ses professeurs, à son entourage. Celui qui s'exprime par le langage du drame fait un tout autre aveu. Le Poëte de L'Endormie fuit la vue de la Femme. Le Frère d'Une Mort prématurée choisit les ténèbres extérieures parce que Dieu n'y est pas. Toujours, dans les premiers drames l'homme fuit la Femme jusqu'à la faire périr. Le refus est profond, viscéral, métaphysique.

Claudel, dans ses Mémoires improvisés, rappelle l'intuition de Baudelaire selon laquelle tout amour est un vase de fiel. Il remarque que, de la même façon, il avait vécu en imagination son drame avant de le vivre dans la réalité. Avant même la rencontre de Rosalie il en avait imaginé, dans La Ville, le scénario : la double tromperie de la femme qui provoque la mort de l'époux avant de trahir son amant après lui avoir donné un fils et se voir finalement exclue de la cité. Tout se passe donc comme si l'événement ne faisait que justifier, actualiser cette fiction qui exprimait une tendance profondément enracinée dans le psychisme de l'auteur. 
Le langage du drame n'a donc rien de gratuit. Il exprime une vérité que le créateur a besoin et peur à la fois d'extérioriser et qu'il ne peut dire que dans cet état second que nous décrivait Cœuvre. Et si Claudel a si longtemps refusé la représentation de Tête d'Or et du Partage de Midi parce qu'il aurait le sentiment d'être nu sur la scène, c'est qu'il n'était pas prêt, sans doute à recevoir cette vérité.

Dans tous les cas, ce langage est mythique. Dans les premiers drames, les personnages, l'action le sont. Dans Partage de Midi, où ils correspondent à une réalité anecdotique, l'auteur fait appel aux grands mythes de l'humanité.

Tête d'Or, dans la première version de son drame, avait bénéficié d'une double élection, celle du sacré d'abord, les dieux, le printemps dionysiaque, celle du divin ensuite, un être personnel qui le prend aux cheveux. Mais il n'avait pu, ou voulu assumer que la première rencontre.

Mesa, lui, est le premier héros de ce théâtre à s'unir au divin. C'est en cela que réside la principale originalité de Partage de Midi qui jusque-là s'inscrivait dans la continuité du théâtre antérieur. A ce moment aussi le langage se modifie. Le mythe cesse d'être traditionnel pour devenir proprement claudélien, celui de la nuit cosmique et du ciel étoile, tel qu'on le retrouvera plus tard, notamment dans Le Soulier de satin. Et, si l'on veut christianiser la pièce, on peut le faire en cela, me semble-t-il, et en cela seulement : c'est le triomphe de la Foi alors que Mesa a toutes les raisons de douter, le triomphe de l'Espérance alors que s'impose le désespoir. Cette affirmation contrebalance, en quelque sorte, l'aveu sans grande humilité d'être incapable de la plus grande vertu chrétienne, la charité, puisque Mesa désire Dieu plus qu'il ne l'aime, est reçu par l'armée des saints plus qu'il ne se donne, et préfère indéfiniment le désir de la femme à sa possession. Et nous avons un aveu proche de la confession directe. Mais le désir à la fois charnel et spirituel et le retrait, l'incapacité de se donner sont vécus sur des plans totalement différents.

\section{NOTES}

1. La Ville, $2^{\text {ème }}$ vers., Pl., Th., 1, 428. Ly exprime la même pensée, $1^{\text {ère }}$ vers., ibid., 311.

2. Partage de Midi, ibid., 984.

3. Ibidem.

4. Ibidem, 987.

5. Ibidem, 988.

6. Ibidem, 1052.

7. Ibidem, 996.

8. Ibidem, 1022.

9. Ibidem, 1025.

10. Ibidem.

11. Ibidem, 1020.

12. Ibidem, 1024.

13. Ibidem, 1014.

14. Ibidem, 1027. 
15. Ibidem, 1042.

16. Ibidem, 1049 .

17. Ibidem, 1050 .

18. Ibidem, 1062 .

19. "Qu'a-t-il donc à me reprocher? parce qu'il ne s'est pas donné, et moi je me suis retirée », Ibidem, 1041

20. Il fallait tout donner,

Et c'est cela que tu n'as pas pardonné, ibidem, 1045.

21. « Et parce que j'ai été un égoïste, c 'est ainsi que vous me punissez

Par l'amour épouvantable d'un autre!»

Ibidem, 1051.

22. Ibidem, 1054

23. Et sache que je en veux pas même de ta présence,

Si elle doit m'arrêter sur moi-même!

Et de ta complaisance, si elle est une limite

A ma fuite hors de cette personne détestée !

Et si le désir devait cesser avec Dieu,

Ah, je l'envierais à l'Enfer !

Fausta : « Cantique du cœur dur », La Cantate à trois voix, Pl., Po., 362. 